\title{
CONF-960813--2 SAND96-1684C \\ IN SITU PRE-GROWTH CALIBRATION USING REFLECTANCE AS A CONTROL STRATEGY FOR MOCVD FABRICATION OF DEVICE STRUCTURES
}

William G. Breiland, Hong Q. Hou, Herman C. Chui*, and Burrel E. Hammons

Sandia National Laboratories, Albuquerque, NM 87185-0601

proofs to:

W. G. Breiland

Dept 1126, MS0601

Sandia National Laboratories

Albuquerque, NM 87185-0601

wgbreil@sandia.gov

(505)844-7029 FAX: (505)844-3211

PACS: 7.60.Hv, 81.15.Gh, 85.60-q

Keywords: MOCVD, reflectance, in situ, virtual interface, VCSEL

RECEIVED

JUL 301996

OSTI

\begin{abstract}
In situ normal incidence reflectance, combined with a virtual interface model, is being used routinely on a commercial MOCVD reactor to measure growth rates of compound semiconductor films. The technique serves as a pre-growth calibration tool analogous to the use of RHEED in MBE as well as a real-time monitor throughout the run. An application of the method to the growth of a vertical cavity surface emitting laser (VCSEL) device structure is presented. All necessary calibration information can be obtained using a single run lasting less than one hour. Working VCSEL devices are obtained on the first try after calibration. Repeated runs have yielded $\pm 0.3 \%$ reproducibility of the Fabry-Perot cavity wavelength over the course of more than 100 runs.
\end{abstract}

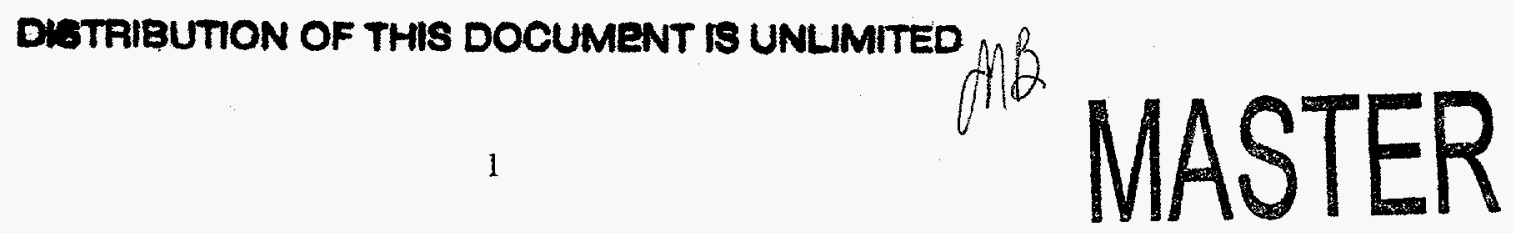




\section{DISCLAIMER}

This report was prepared as an account of work sponsored by an agency of the United States Government. Neither the United States Government nor any agency thereof, nor any of their employees, makes any warranty, express or implied, or assumes any legal liability or responsibility for the accuracy, completeness, or usefulness of any information, apparatus, product, or process disclosed, or represents that its use would not infringe privately owned rights. Reference herein to any specific commercial product, process, or service by trade name, trademark, manufacturer, or otherwise does not necessarily constitute or imply its endorsement, recommendation, or favoring by the United States Government or any agency thereof. The views and opinions of authors expressed herein do not necessarily state or reflect those of the United States Government or any agency thereof. 


\section{DISCLAIMER}

Portions of this document may be illegible in electronic image products. Images are produced from the best available original document. 


\section{Introduction}

The fabrication of semiconductor thin films using chemical vapor deposition (CVD) has traditionally been accomplished by introducing reactive gases into a chamber using timed sequences for a fixed set of reactor conditions. Temperatures, pressures, and flow rates are determined in advance by performing calibration runs before an actual device structure is produced. The results of these calibration runs are used in an iterative "dead reckoning" control strategy to make adjustments in the device structure recipe. Unfortunately, calibration runs must be performed whenever a new chemical source is added, equipment is replaced in the gas handling system, or a new device structure is to be grown. All reactors also experience drifts over time periods ranging from days to weeks, requiring re-calibration. Most calibrations are performed first with test structures and finally with repeated trials on the actual device structure using post-process ex situ analysis tools such as microscopy, photoluminescence, and X-ray diffraction methods. It is often very difficult to determine the origin of a failure or process deviation using postgrowth analysis during the final fine tuning of the device structure recipe. The grower is often forced to guess what portion of the recipe needs alteration and then test this guess with another growth run. A large portion of the time and expense of thin film CVD is thus spent in calibration and post-process failure analysis.

In this paper we address the above deficiencies with a pre-growth calibration method that uses normal incidence reflectance. In many respects the method assumes the same 
role as reflection high-energy electron diffraction (RHEED) does in molecular beam epitaxy. The method can be viewed as an interim step between the traditional postprocess analysis approach and real-time process control. The instrumentation is simple, inexpensive, and robust. Its use greatly simplifies the pre-growth calibration process by allowing one to measure the growth rate from each source gas in a single, relatively short deposition run. The reflectance probe also serves as a valuable real-time diagnostic of the state of the wafer throughout the device structure growth.

With further development, real-time process control of thickness and composition using reflectance appears to be feasible. However, it is important to note that we have found the control strategy described in this paper to be completely adequate for growing even complex device structures such as vertical-cavity surface emitting lasers (VCSEL's). If the run-to-run behavior of the CVD reactor is sufficiently stable over the time period of a few days, very little would be gained by attempting to adopt a real-time control strategy.

The paper is divided into two sections. The first section describes the theory behind the calibration method and details of the data analysis. The second section illustrates the practical use of the method with an example from the growth of a VCSEL structure.

\section{Virtual Interface Analysis of Normal Incidence Reflectance}

\subsection{THE VIRTUAL INTERFACE CONCEPT}


It is straightforward to model the reflectance from a smooth semiconductor substrate with an arbitrary number of smooth, homogeneous films deposited on it $[1,2]$. The only parameters required are the complex refractive index for the substrate and the thickness and refractive index of each layer. If a growing film is monitored, reflectance interference oscillations are observed as the topmost film thickness changes with time. This is also straightforward to model by expressing the topmost film thickness as the product of a growth rate and the time.

There are, however, severe practical limitations that arise when such a straightforward approach is used to model the in situ reflectance waveform of a growing multiple-layer film. First, the deposition is performed at elevated temperatures. The optical constants of most semiconductor materials are not well known at the temperatures typical for CVD growth. This is particularly true for compound semiconductor alloys. Second, the thickness of a layer is not necessarily known precisely, particularly during a calibration run. In a multiple-layer reflectance model, errors in the refractive index and thickness of each layer contribute additively to uncertainties in the description of the growth of the topmost layer. This leads to a growth rate determination that is progressively less accurate with each additional calibration layer.

The solution to the multiple-layer reflectance modeling problem is to use a virtual interface concept, which is illustrated in Fig. 1. One chooses a virtual interface position (dashed line) that lies anywhere within the topmost film. It is then possible to rigorously describe the effects of all underlying layers as a single effective complex refractive index, 
$N_{v i}$, of a virtual interface effective substrate. The precise value of $N_{v i}$ can, in principle, only be calculated from a complete knowledge of all the refractive indexes and thicknesses of the underlying layers. However, if $N_{v i}$ is taken to be an unknown, it is always true that any multiple-layer structure requires only two parameters, the real and imaginary parts of $N_{v i}$, to describe the effects of all underlying layers below the virtual interface boundary. Analysis of the topmost layer is thus made completely independent of the optical constants and interface positions of underlying layers. By choosing a new virtual interface position with each new layer, cumulative effects are eliminated in the analysis of a growing multiple-layer film structure. Aspnes [3] first suggested the use of a virtual substrate in the analysis of in situ ellipsometry of growing thin films. A slightly modified version of this concept was applied to normal incidence reflectance by Breiland and Killeen [4]. In what follows, we briefly review the virtual interface formalism as presented in ref. [4], with particular emphasis on its practical implementation as a pre-growth monitor. A full derivation and discussion of the theory behind this method is given in ref. [4].

The time-dependent normal incidence complex reflectance, $r(t)$, of a multiple-layer growing film may rigorously be described with the following equation:

$$
r(t)=\frac{r_{\infty}+r_{v i} \exp (-i 4 \pi N G t / \lambda)}{1+r_{\infty} r_{v i} \exp (-i 4 \pi N G t / \lambda)}
$$


The film thickness is expressed as the growth rate, $G$, times the time, $t$, where the virtual substrate interface is defined to be at $t=0 . \quad r_{\infty}$ is the complex reflectance of an infinitely thick film of the topmost layer and is given explicitly by $(1-N) /(1+N)$, where $N$ is the complex refractive index of the topmost growing film, $N=n-i k$, at the wavelength, $\lambda . r_{v i}$ is the virtual interface complex reflectance just inside the topmost layer and is the ratio of backward and forward electric field amplitudes at the virtual interface boundary. It is formally related to the virtual interface effective refractive index by $r_{v i}=\left(N-N_{v i}\right) /\left(N+N_{v i}\right)$. For the purposes of the pre-growth calibration method, $r_{v i}$ is simply treated as an adjustable parameter in a least-squares fit to an observed reflectance waveform, and $N_{v i}$ is never calculated.

Several features of eq. (1) make it particularly suitable for analyzing growing films. The expression contains five materials parameters: the growth rate, $G$, and the real and imaginary parts of $N$ and $r_{v i}$. These parameters are linearly independent and can thus be determined with a least-squares fit to an observed reflectance waveform, $R(t)=|r(t)|^{2}$. The frequency of the reflectance oscillation is determined by the product, $N G$. If the absolute reflectance is recorded, the $r_{\infty}$ term in eq. (1) effectively provides a measure of $N$ independent of $G$, allowing one to separate the $N G$ product. It is thus possible to determine the growth rate of a thin semiconductor film with in situ normal incidence reflectance without having to know the refractive index of the film during growth conditions. 
The accuracy of $G$ is related directly to the accuracy of the absolute reflectance. A one percent error in reflectance results in approximately one percent error in growth rate. It is thus very important to self-calibrate the reflectance at the beginning of each run and to maintain instrument stability throughout the run.

With ideal signal-to-noise $\left(10^{4}\right)$, it is possible to obtain one percent accuracy in the growth rate with a film thickness on the order of $\lambda / 4 n$. Accuracy improves exponentially with film thickness up to $\lambda / 2 n$, at which point it becomes noise limited. These thicknesses may be excessive if one is considering real-time control of thin structures, but there is no such limitation on the thickness of a pre-growth calibration film.

\subsection{GROWTH RATE EXTRACTION}

Reference [4] outlines a method by which one can use a reflectance waveform having at least several extrema of oscillations to estimate the values of the parameters to be fit [5]. This becomes extremely useful for routine pre-growth calibrations. The calibration procedure is as follows: A multiple-layer film is grown and a single-wavelength in situ reflectance interferogram is recorded. Each layer is grown thick enough to provide several extrema of interference oscillations. The order of the layers and timing is chosen to yield good contrast between the interferograms in each layer. For example, a GaAs growth calibration layer is done after an AlAs calibration layer is placed on the GaAs substrate. Segments of data from each layer are chosen for analysis. The starting time for each 
segment is arbitrary, provided that it does not include the transition from one layer to the next. The choice of starting time changes only the value of $r_{v i}$, which is of no physical interest. Typically, the starting segment is chosen to be several seconds beyond the time at which the analyzed layer is known to have started. The stopping time is chosen to be a few seconds before the next layer is known to have started. The automated procedure outlined in ref. [4] is used to provide starting estimates for the five-parameter fit. A least-

squares analysis is then done to fit the data segment to $R(t)=|r(t)|^{2}$, eq. (1), and provide the growth rate for the layer. This can all be accomplished without prior knowledge about the growth rates or optical constants of the deposited materials. The process is then repeated for each layer in the calibration run. Fitting takes no more than a few seconds for each layer.

\section{Application to the growth of VCSEL's}

In this section, we illustrate the application of the virtual interface method described above to the growth of an optoelectronic device structure. We choose the fabrication of a vertical cavity surface emitting laser (VCSEL) as an example of an extremely complex structure that requires many layers of differing alloy composition and very precise thickness control. The strategies used in this structure provide a general example of how the method may be implemented in other applications. 


\subsection{APPARATUS}

Normal incidence reflectance requires only one window, is insensitive to incidence angle, (allowing one to monitor "wobbling" substrates) and is also insensitive to light polarization. It is therefore simple, robust, and well suited to a production environment. The apparatus is shown in Fig. 2. It is a non-intrusive, compact assembly, measuring only $15 \mathrm{~cm}$ in its longest dimension, that mounts directly on the top window of the reactor. A 7 watt tungsten halogen lamp is used for the light source. This lamp is chosen because it is small, inexpensive, more stable in amplitude than most lasers, and provides a continuous range of wavelengths from $350 \mathrm{~nm}$ to several microns. The lamp illuminates a $0.5 \mathrm{~mm}$ aperture which is imaged as a $2.5 \mathrm{~mm}$ spot on the wafer using a $15 \mathrm{~mm}$ diam., $50 \mathrm{~mm}$ focal length lens. The light is directed through a $3 / 4$ in. diam. purged window above the wafer carrier in a commercial EMCORE 3200 rotating disk CVD reactor. Reflected light is deflected with a beam splitter, imaged on a $0.6 \mathrm{~mm}$ aperture, and detected with a silicon photodiode placed behind a $10 \mathrm{~nm}$ bandwidth interference filter. The current from the photodiode is fed to a transimpedance amplifier and the output is digitized with an $A / D$ board in a personal computer. A monitor photodiode with an identical interference filter is also mounted on the beam splitter. The reflected light signal is divided by the monitor signal to cancel out fluctuations and drifts in the lamp source. Window and interference filter surfaces must be tilted slightly to eliminate spurious reflections. Spectral reflectance 
may be implemented with this apparatus by replacing the $0.6 \mathrm{~mm}$.aperture with a $0.6 \mathrm{~mm}$ optical fiber connected to a spectrograph [6].

The optical arrangement in Fig. 2 serves several purposes. 1) It provides a means by which a relatively small spot of light may be imaged on the surface of the substrate using a highly divergent lamp source. 2) It relays the image of the first aperture to the surface of the wafer and then to the receiving aperture. The spot on the substrate thus acts as an effective source for the second lens. Due to the conjugate relationship between the spot on the substrate and the receiving aperture, small shifts in the position and tilt of the substrate do not significantly affect the flux of light passing through the receiving aperture. 3) It acts both as a spatial filter as well as a wavelength filter. Light originating from the much brighter $3000 \mathrm{~K}$ lamp source completely overwhelms the $\sim 1000 \mathrm{~K}$ thermal radiation from the spot on the heated substrate viewed by the receiving aperture. This allows one to directly record the DC signal without chopping or lock-in detection. Current to voltage conversion with the transimpedance amplifier is performed in a photovoltaic mode without reverse bias on the silicon photodiode. This provides a linear signal with a drift-free, truezero baseline and negligible dark current.

Absolute reflectance is obtained by referencing the starting signal to the known reflectance of the substrate before deposition begins. This provides a self-calibration at the beginning of each growth run.

GaAs and $\mathrm{Al}_{x} \mathrm{Ga}_{1-x} \mathrm{As}$ layers were typically grown at $750{ }^{\circ} \mathrm{C}$ by using trimethylaluminium (TMA), trimethylgallium (TMG), and 100\% arsine $\left(\mathrm{AsH}_{3}\right)$. The alkyl 
sources carried by high-purity $\mathrm{H}_{2}$ were injected into the reactor through 3 different injection zones distributed along the radial direction of the top flange. The Gas-flow partitioning for alkyl sources among these three injection zones was set at 7\%:78\%:15\% between the inner, middle, and outer zones to give the best thickness uniformity across the wafer. The substrate was rotated at $1000 \mathrm{rpm}$ at a pressure of 60 torr. Group V flow and total reactor flow were $248.5 \mathrm{sccm}$ and $32.6 \mathrm{slm}$, respectively.

\subsection{THIN FILM REQUIREMENTS FOR VCSEL GROWTH}

Our VCSEL device consists of over 600 layers of $\mathrm{Al}_{\mathrm{x}} \mathrm{Ga}_{1-\mathrm{x}} \mathrm{As}$ alloys. The composition, $x$, of the alloy is piece-wise linearly graded to emulate a parabolic composition profile in the layers that comprise the distributed Bragg reflector (DBR) stacks. The bottom mirror stack is doped n-type and the top stack is doped p-type. The n-type doping poses no problems, but p-type doping is achieved using $\mathrm{CCl}_{4}$ as a source gas. The chlorine in this source causes an etchback reaction to occur that alters the overall deposition rate of the doped $\mathrm{Al}_{x} \mathrm{Ga}_{1-\mathrm{x}} \mathrm{As}$ alloy. The etchback rate is a function of alloy composition and growth temperature, which complicates the growth of the graded alloy layers. Two deposition temperatures are required for successful device performance. Because the cavity region of the device may require very different growth rates than the DBR stacks, two sources of $\mathrm{Ga}$ and two sources of $\mathrm{Al}$ are necessary to avoid pauses in the 
growth recipe. Calibration of such a complicated structure is formidable using traditional post-process calibration methods.

After some preliminary reflectance experiments to investigate the growth behavior of the system, it was determined that a VCSEL structure could be calibrated with a single run. Below is a summary of the preliminary experiments, followed by a description of the final calibration procedure.

\subsection{GENERAL AlAs and GaAs GROWTH BEHAVIOR}

A growth run was performed to determine the overall behavior of the reactor during the deposition of AlAs and GaAs. This run is shown in Fig. 3. Alternating layers of AlAs and $\mathrm{GaAs}$ were grown at $750^{\circ} \mathrm{C}$, each layer having a different source gas flow rate. The different growth rates that result from this experiment are readily apparent as different oscillation frequencies. Fig. 4 shows the virtual interface analysis of the data in Fig. 3 . Each data point represents a virtual interface analysis from one segment of data in Fig. 3 . The growth rates are seen to be linear in source gas flow rate, as would be expected in a diffusion-limited CVD reaction. The composition, $x$, of $\mathrm{Al}_{\mathrm{x}} \mathrm{Ga}_{1-\mathrm{x}}$ As alloys is linearly related to the relative growth rates of AlAs and GaAs. These two linear features greatly simplify the task of growing a VCSEL structure in which the DBR stacks must be graded in composition. A complete calibration requires only the determination of the growth rate of AlAs and GaAs at one flow rate. This measurement is sufficient to determine the 
slopes of the lines in Fig 4. From this, one may calculate all necessary flow rates for a series of graded-composition $\mathrm{Al}_{\mathrm{x}} \mathrm{Ga}_{1-\mathrm{x}}$ As alloy layers.

Fig. 4 also reveals that the TMG flow controller has a small but significant zero offset. A high-precision tool such as the reflectance probe is needed to reveal this kind of deviation.

\subsection{ETCHBACK GROWTH BEHAVIOR}

A calibration run similar to that of Fig. 3 was performed for a range of alloy compositions, with and without doping. From the virtual interface analysis of the growth rates, calibration functions were constructed to allow one to compensate for etchback effects at any composition. As with the general calibration above, it was determined that only one etchback measurement needed to be performed to completely determine flow rates needed to produce p-type DBR's.

\subsection{SPECIFIC CALIBRATION SCHEME FOR 845 nm AND 770 nm VCSEL's}

After the background work outlined above, it was determined that a single calibration run involving only six layers is necessary to completely characterize the system for growth of a VCSEL centered at either $845 \mathrm{~nm}$ or $770 \mathrm{~nm}$. The reflectance interferogram from such a calibration is shown in Fig. 5. The run determines the $750{ }^{\circ} \mathrm{C}$ growth rates from 
the first $\mathrm{Al}$ source, the first $\mathrm{Ga}$ source, the second $\mathrm{Al}$ source, the second $\mathrm{Ga}$ source, and the etchback rate when $\mathrm{CCl}_{4}$ is introduced. Finally, the temperature is dropped to $640{ }^{\circ} \mathrm{C}$ and another growth rate in the presence of doping is determined. In a single run lasting less than one hour, all calibration information is obtained with the in situ reflectance monitor.

The above calibration scheme yields working VCSEL devices without the need for further fine tuning of the system. Because our CVD reactor is very reproducible, only periodic re-calibration is needed between a series of nuns. Fig. 6 shows the results of many VCSEL growths in which the calibration was performed only before each group of runs shown. A good figure of merit for VCSEL's is the reproducibility of the Fabry-Perot cavity wavelength. Excellent reproducibility is obtained, and the target wavelength is achieved with the first device grown after each calibration. Also note that a single $845 \mathrm{~nm}$ run was inserted within the group of $770 \mathrm{~nm}$ VCSEL's. Calibration values from the 770 nm group were used to compute the recipe for the single $845 \mathrm{~nm}$ run. This represents a good example of the agile manufacturing capability of the pre-calibration method.

\subsection{REAL TIME GROWTH MONITOR}

In addition to its use as a calibration tool, the reflectance instrument is used to monitor the growth of every structure. Fig. 7 shows a typical reflectance interferogram of an $845 \mathrm{~nm}$ VCSEL run. It is easy to observe the growth of the buffer layer, $n$-type DBR, 
laser cavity region, $p$-type $\mathrm{DBR}$, and the contact cap layer. Such a monitor is extremely valuable as a real time fault detector, signaling such events as a depleted source or a rough surface. In addition to detecting the presence of a fault, the reflectance history can very often pinpoint the source of the failure. This eliminates the need to perform additional diagnostic growth runs to track down and identify what actually caused the failure.

\subsection{Summary}

In situ reflectance has become an indispensable routine tool for pre-growth calibration and growth monitoring in our laboratory. It has proved to be an efficient and very precise method for calibrating growth rates for the reactor. As a real time monitor, reflectance has saved a great deal of time and expense in detecting and identifying problems during growth. Our experience with VCSEL growth demonstrates that there are opportunities to achieve excellent process control without having to resort to a real time strategy. In the future, we plan to use the full reflectance spectrum, rather than just one wavelength, and to use the refractive index information that is extracted from the virtual interface analysis. Both growth rates and chemical composition of compound semiconductor alloys may then be determined with the spectral reflectance technique. 


\section{Acknowledgments}

This work was funded by the Department of Energy under Sandia National Labs DOE contract DE-AC04-94AL85000.

\section{References}

* Present address: Hewlett Packard Co, 370 W. Trimble Rd., San Jose, CA, 95131.

[1] O. S. Heavans, Optical Properties of Thin Solid Films (Dover, New York, 1965).

[2] R. M. A. Azzam and N. M. Bashara, Ellipsometry and Polarized Light (North Holland, Amsterdam, 1988).

[3] D. E. Aspnes, J. Opt. Soc. Am. A 10 (1993) 974.

[4] W. G. Breiland and K. P. Killeen, J. Appl. Phys. 78 (1995) 6726.

[5] There is a scaling error in the third line of eq. (9) in reference 4 . The line should read: $n \cong \delta \lambda / 4 \pi G, k \cong \gamma \lambda / 4 \pi G$.

[6] K. P. Killeen and W. G. Breiland, J. Electron. Mat. 23 (1994) 179.

\section{Figure Captions}

Fig. 1. Any multiple-layer thin film structure having refractive indexes, $N_{i}$, and thicknesses, $d_{i}$, may always be modeled as a single-layer film on a virtual substrate with an effective refractive index, $N_{v i}$. All the optical effects of the various $N_{i}$ and $d_{i}$ are lumped into the value for $N_{v i}$. The virtual interface need not coincide with an actual interface, but must be somewhere inside the actual topmost film. 
Fig. 2. Schematic of the instrument used to record normal incidence reflectance. A $633 \mathrm{~nm}$ interference filter was used for all the data reported in this paper.

Fig. 3. Reflectance from twelve layers of alternating $\operatorname{AlAs}(2500$ $\AA) / \operatorname{GaAs}(2500 \AA)$ thin films. Different alkyl flow rates were used for each layer.

Fig. 4. Growth rates as a function of alkyl flow rate extracted from the data in Fig. 3 using virtual interface analysis.

Fig. 5. Reflectance interferogram for a six-layer pre-growth calibration run. All information needed to set up a growth recipe for a VCSEL is obtained from this calibration run.

Fig. 6. Reproducibility of the cavity wavelength of $770 \mathrm{~nm}$ and $845 \mathrm{~nm}$ VCSEL structures. A virtual interface calibration was performed before each group of data shown.

Fig. 7. A reflectance interferogram recorded during the complete growth of a $845 \mathrm{~nm}$ VCSEL. 


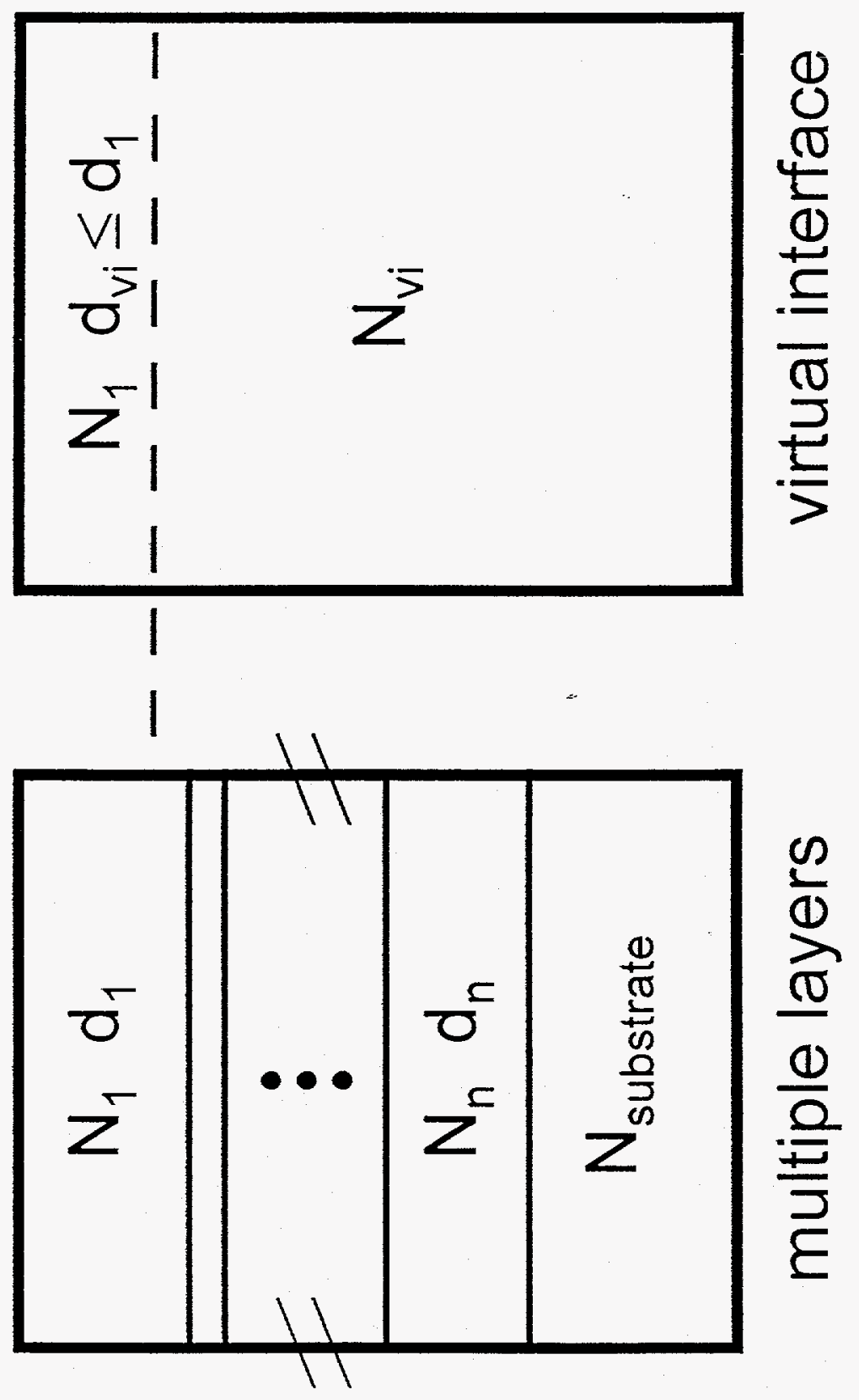




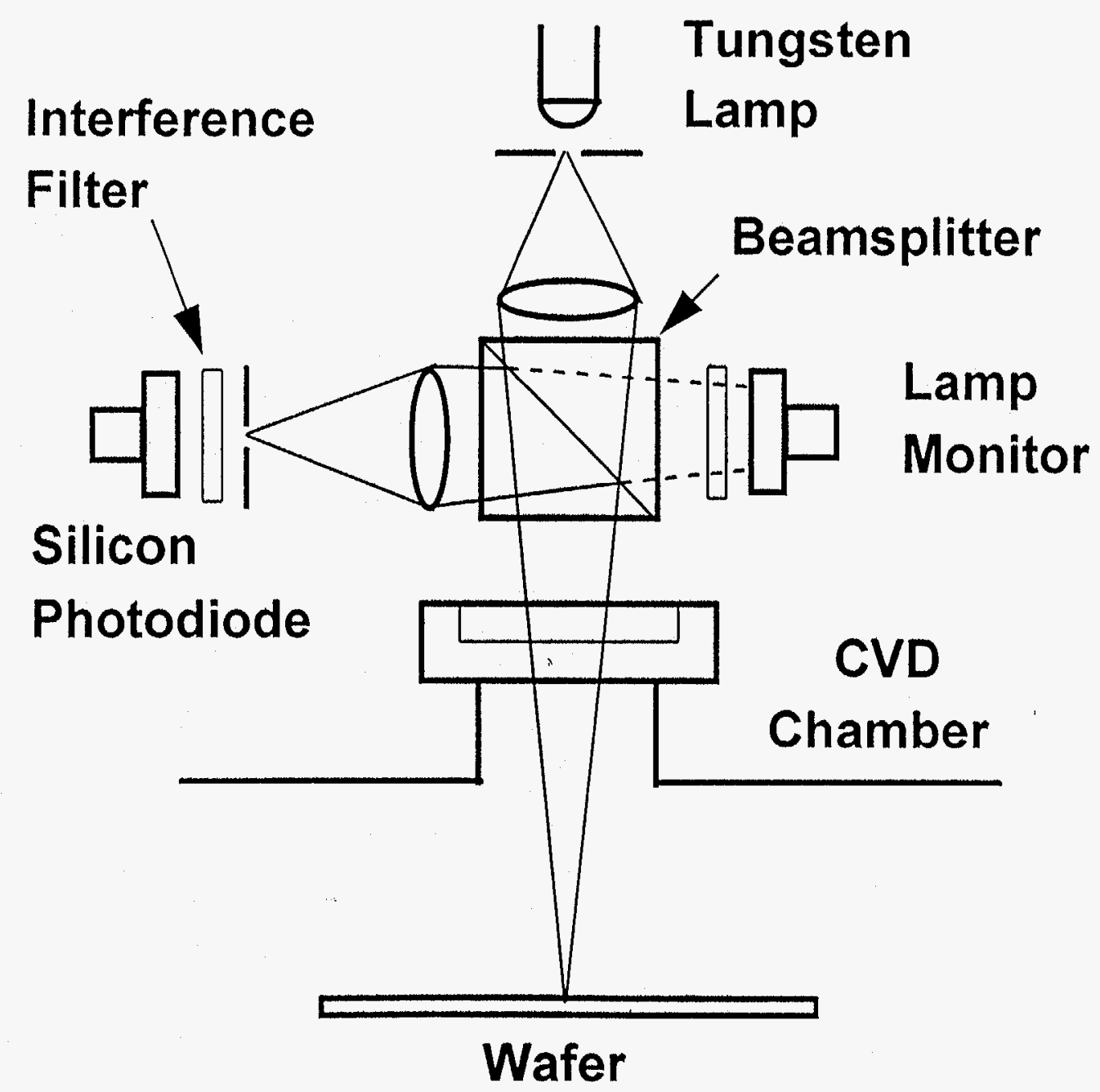

FIG. 2 


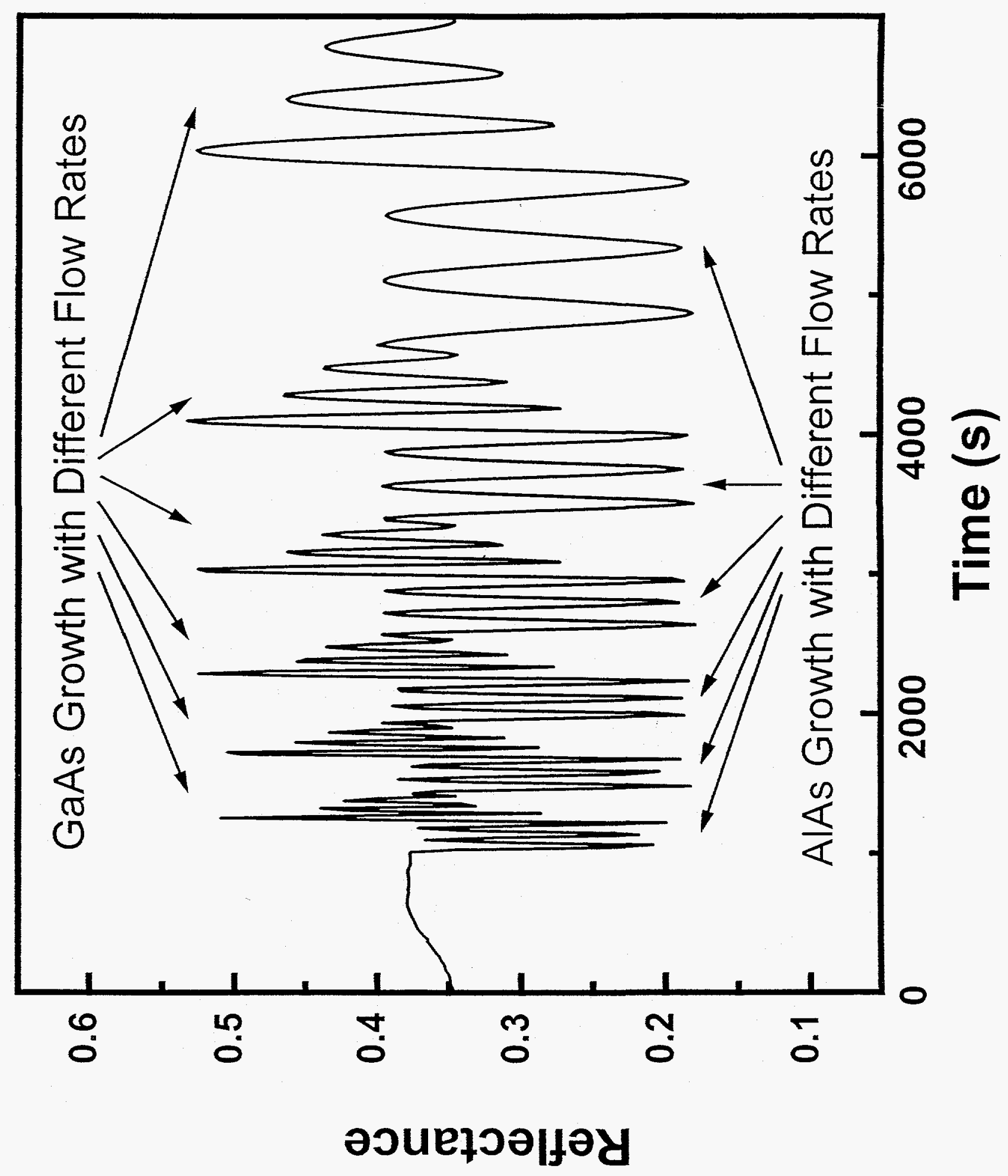




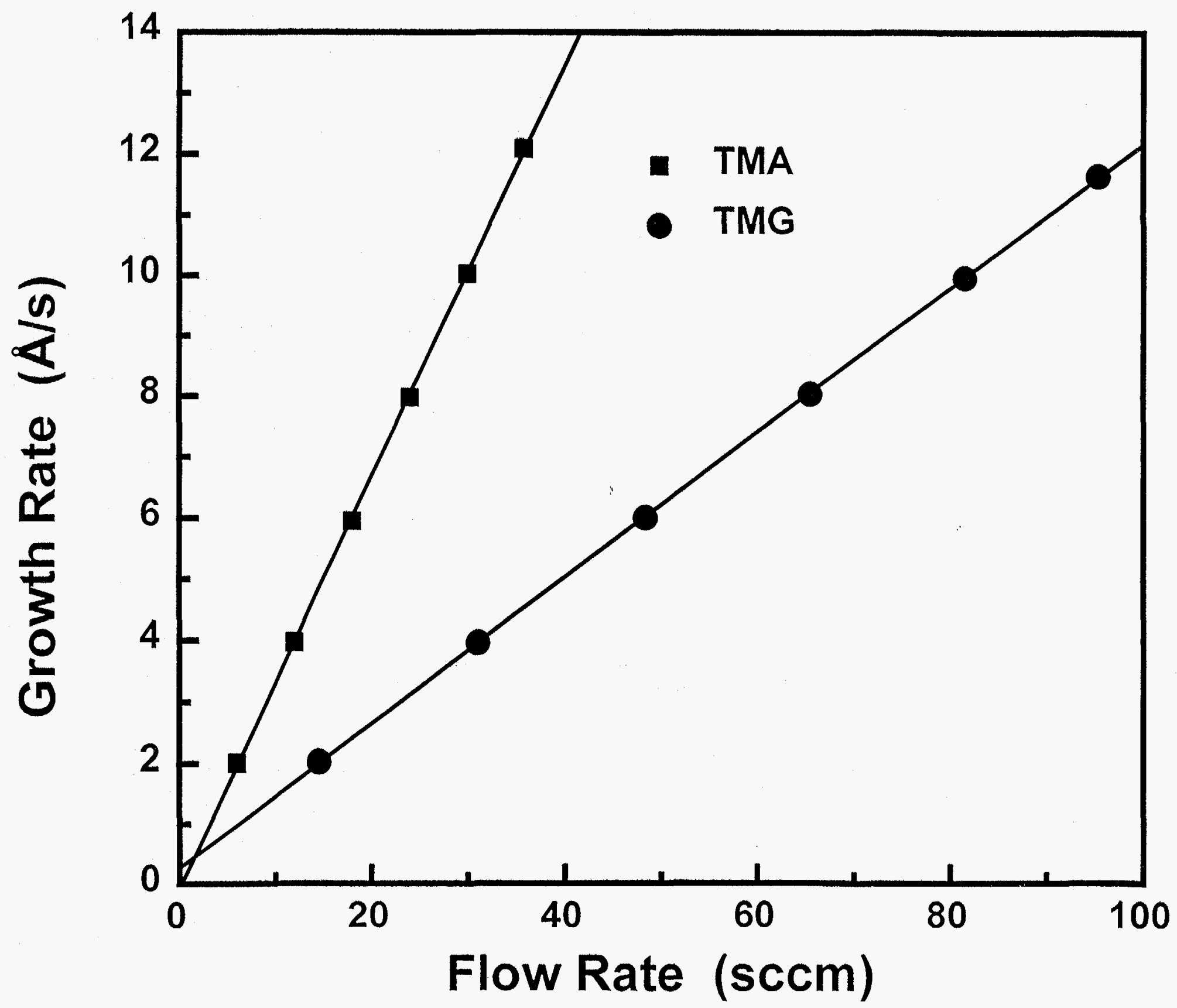




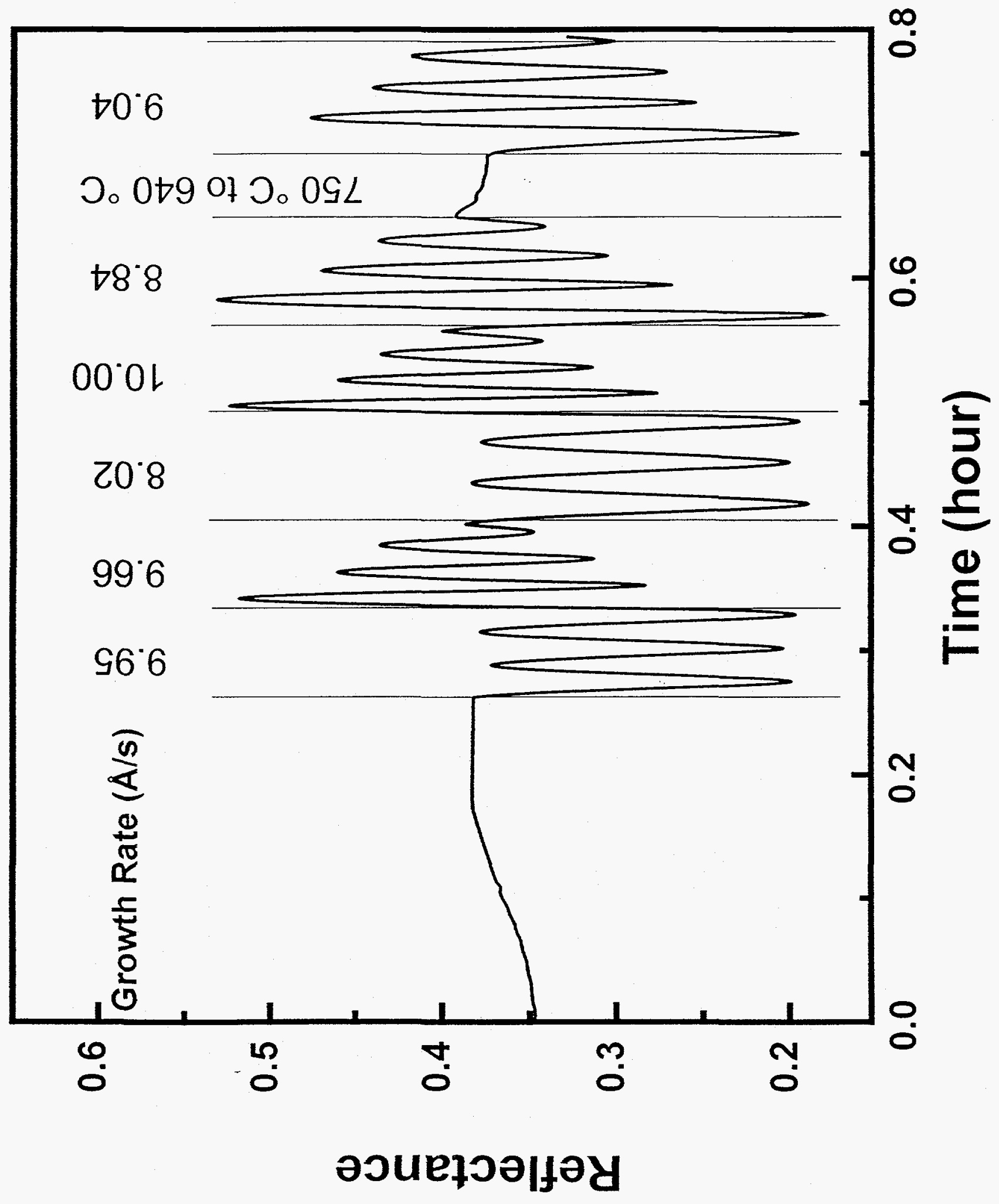




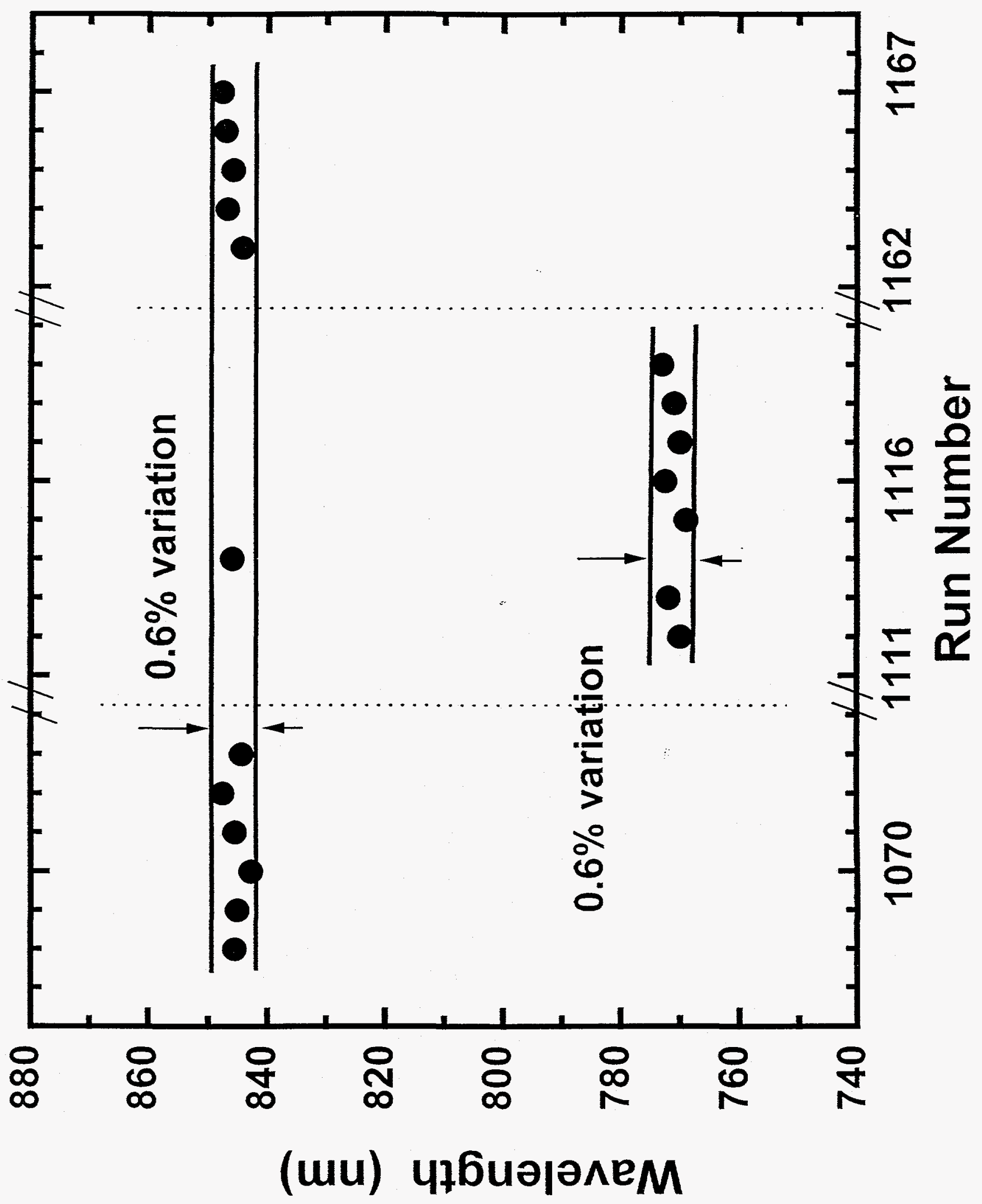




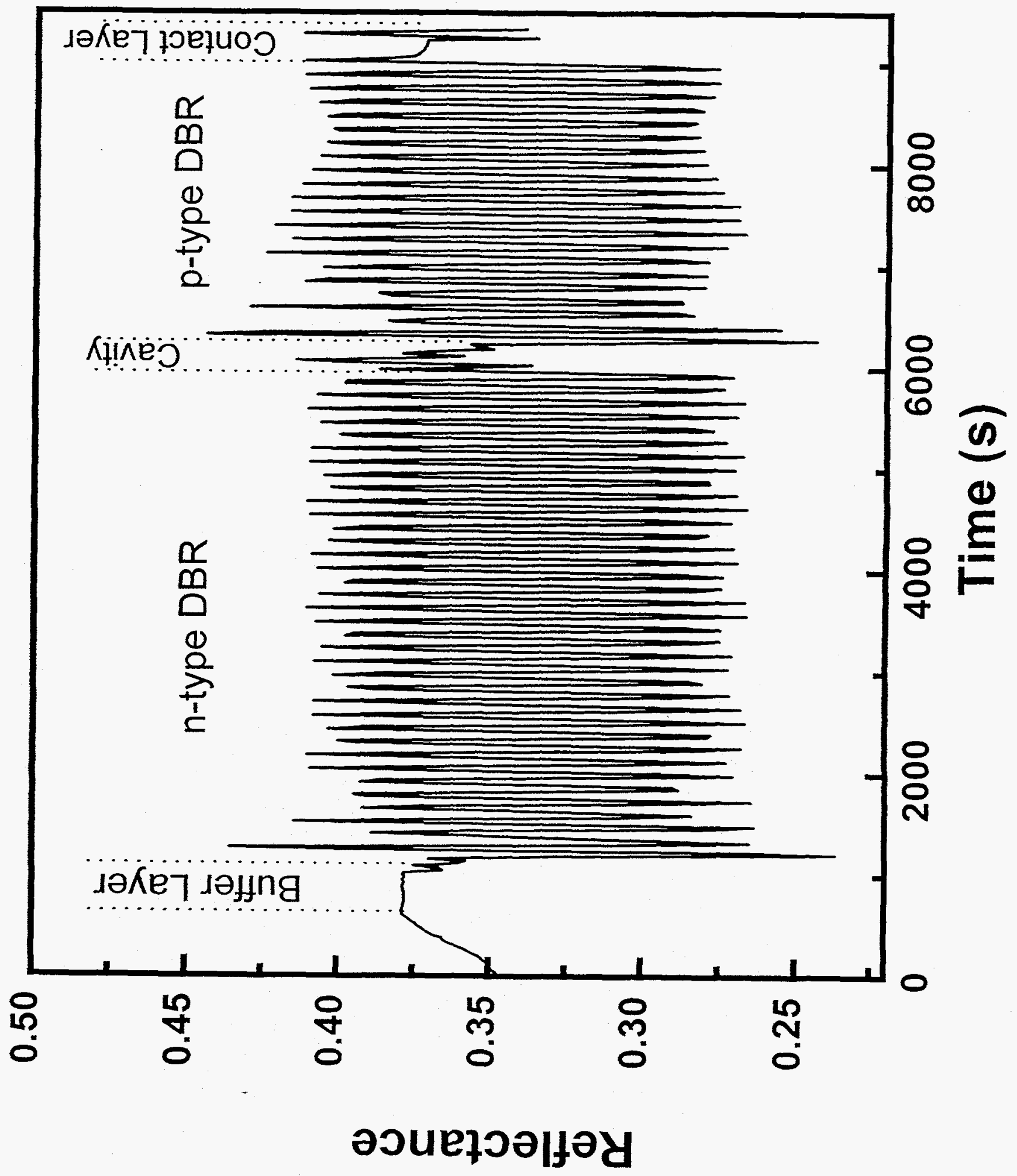




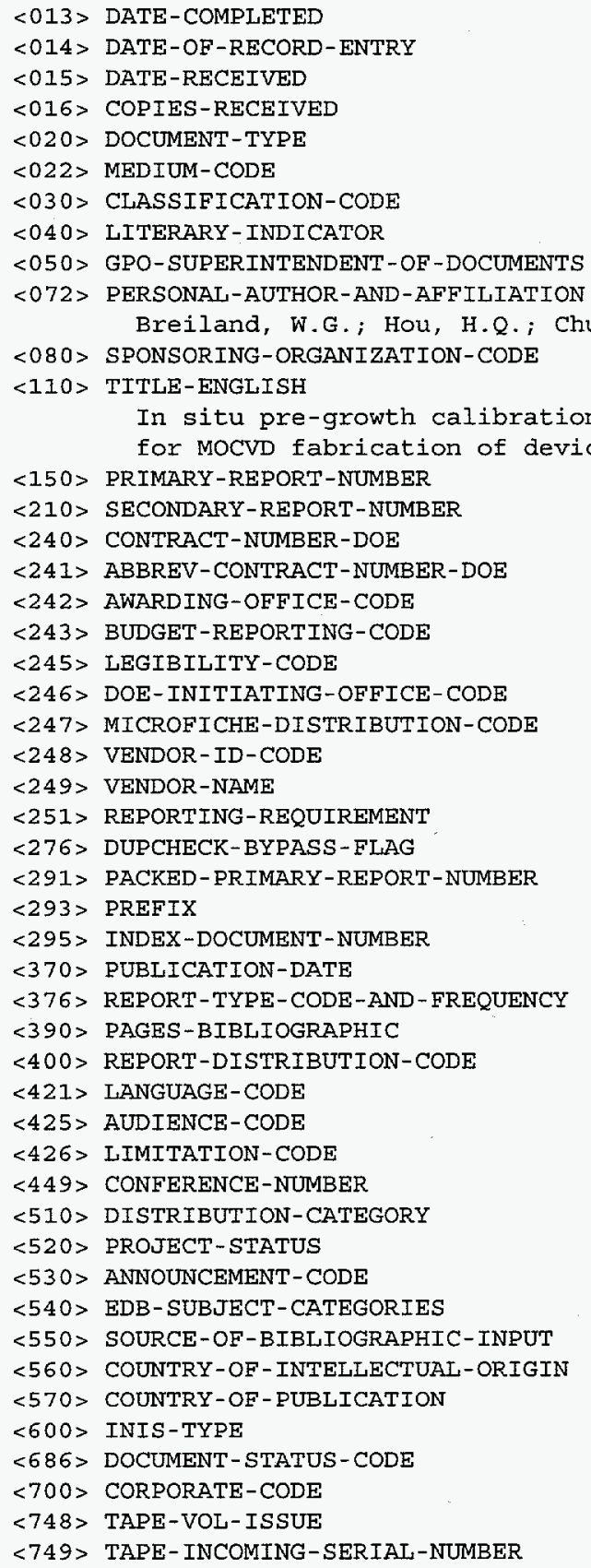

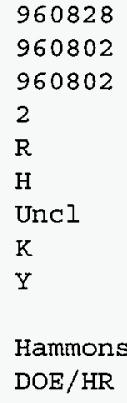

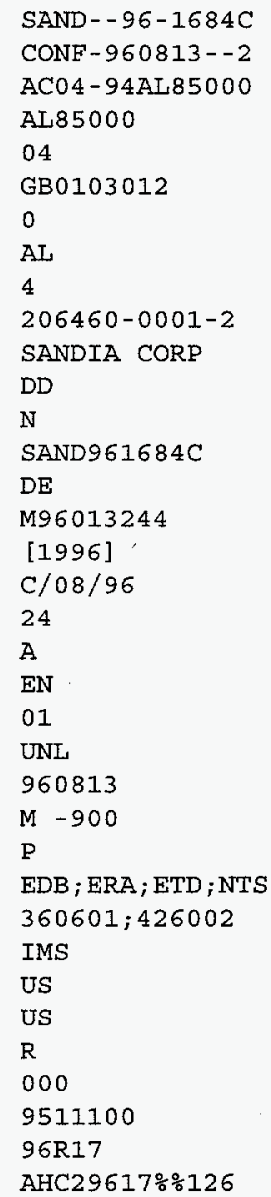




\section{$<801>$ SUBJECT-DESCRIPTORS}

DIAGNOSTIC TECHNIQUES; CHEMICAL VAPOR DEPOSITION:Q1, Q2, T3; CRYSTAL GROWTH; SEMICONDUCTOR MATERIALS:T1;MOLECULAR BEAM EPITAXY;SEMICONDUCTOR LASERS : T2 ; CHEMICAL REACTORS;CALIBRATION;ELECTRON DIFFRACTION; PROCESS CONTROL : $Q 3$

<931> AVAILABILITY-CODE

OS; NT

$<950>$ ABSTRACT

In situ normal incidence reflectance, combined with a virtual

interface model, is being used routinely on a commercial MocvD reactor to measure growth rates of compound semiconductor films. The technique serves as a pre-growth calibration tool analogous to the use of RHEED in MBE as well as a real-time monitor throughout the run. An application of the method to the growth of a vertical cavity surface emitting laser (VCSEL) device structure is presented. All necessary calibration information can be obtained using a single run lasting less than one hour. Working VCSEL devices are obtained on the first try after calibration. Repeated runs have yielded $\left\{t^{-}\right\} 0.3 \%$ reproducibility of the Fabry-Perot cavity wavelength over the course of more than 100 runs. 